Liliana Karina Alanís Flores*

\title{
DICCIONARIOS Y TRADUCTORES EN LÍNEA EN E/LE: UN ANÁLISIS DESDE LA TEORÍA DE LA ARGUMENTACIÓN EN LA LENGUA
}

\begin{abstract}
En este artículo se defiende la tesis de que la teoría de la argumentación en la lengua puede ser aplicada tanto en la teoría como en la práctica de la enseñanza de español como lengua extranjera. Especialmente, esta teoría puede ayudar a explicar las deficiencias que aún presentan las herramientas de traducción y diccionarios online así como las ventajas que representa su uso en el desarrollo de las competencias de los estudiantes en el nuevo idioma. En el trabajo se hace énfasis en que, ya que el centro de la enseñanza de segundas lenguas es en la actualidad la competencia comunicativa, los profesores deben propiciar al mismo tiempo el desarrollo de la competencia pragmática en los estudiantes, de la misma manera como los estudiantes deben tener una preparación que les permita usar estas herramientas tecnológicas de manera más efectiva. El artículo presenta algunos ejemplos de uso del traductor de Google y del diccionario Linguee para fundamentar estas tesis.
\end{abstract}

Palabras clave: español como lengua extranjera, pragmática, análisis del discurso, marcadores discursivos, argumentación en la lengua, competencia comunicativa

\section{Introducción}

Las tendencias más recientes en el ámbito de la enseñanza del español como idioma extranjero se basan en un enfoque comunicativo (Communicative Language Teaching), dentro del cual, el foco principal es desarrollar la competencia comunicativa del hablante en el nuevo idioma, colocando a la gramática dura en una posición secundaria, pues lejos de ser el centro del aprendizaje, se le trata como un medio con la que el estudiante podrá comunicarse y desenvolverse en un contexto lingüístico determinado. Este enfoque comunicativo ha puesto en evidencia que, además del código, es

karina.alhaniz@gmail.com 
necesaria una competencia pragmática y discursiva para poder llegar a la comunicación efectiva en las situaciones más cotidianas:

A theory of language as communication lies at the very core of the CLT. Hymes (1972) advanced the notions of «competence» and «performance» introduced by Chomsky in the 1960s and stated that the goal of language teaching was to develop «communicative competence», which implied acquiring both an ability and knowledge to use language. In other words, communicative competence considers language as a tool used for communication. (Basta, 2011: 126)

Siguiendo esta línea, es fundamental que el estudiante de español como lengua extranjera (E/LE), durante el proceso de desarrollo de estas competencias, tome conciencia también de que su aprendizaje debe orientarse a interactuar exitosamente en un determinado contexto social, ya que esto es uno de los preceptos principales de una enseñanza centrada en el desarrollo de las competencias comunicativas:

The communicative ability important for participation in academic, professional and social settings comes with practice, practice along with critical and self-critical analysis of language use. Talking about communication involves talking about grammar, yes, and more. Knowledge of language includes knowledge of grammar, syntax, vocabulary, modes of discourse, print and nonprint genres, and rhetorical strategies, the use of language to influence others. Learner metalinguistic and metacognitive awareness begins with awareness of self and of the ways in which one can mean. (Savignon, 1997: 256)

Por esta razón, el enfoque comunicativo se impone en la actualidad en las aulas, y en consecuencia, se vuelve una necesidad para los profesores construir nuevas estrategias para la enseñanza de la lengua extranjera. Una tarea importante es la de enfatizar en el aprendiz la relevancia de los marcadores discursivos, que por su carácter instruccional en la lengua, son fundamentales para que el estudiante pueda comprender, expresar y procesar de mejor manera la información que comparte con otros hablantes de la lengua española. Manuel Martí Sánchez define estas partículas lingüísticas de la siguiente manera:

Los marcadores son un conjunto variado de palabras y expresiones cuya misión siempre debe entenderse en relación con las circunstan- 
DICCIONARIOS Y TRADUCTORES EN LÍNEA EN E/LE: UN ANÁLISIS DES DE LA TEORÍA DE LA ARGUMENTACIÓN EN LA LENGUA

cias en que aparece el enunciado [...] La misión de los marcadores es doble. Por un lado, sirven para enlazar su enunciado con el anterior. Por otro lado, los marcadores son guías fundamentales para poder decir lo que está diciéndose" (2003: 7).

El mismo autor, afirma que, una de las pautas para reconocer los progresos del estudiante en el dominio de la lengua, es el uso fluido y efectivo de los marcadores discursivos:

Por un lado los marcadores discursivos se utilizan en la construcción de estructuras complejas que requieren competencia pragmática y discursiva desarrollada. Por otro, no sorprende que su incorporación a la competencia de los hablantes extranjeros se deba más que al aprendizaje en un contexto académico, a la adquisición ligada al contacto diario con la lengua hablada. (2008: 9)

La pragmática y el análisis del discurso como perspectivas de análisis dentro de la lingüística, nos son de gran utilidad justamente para mostrar el importante rol de los marcadores discursivos en el proceso enseñanza-aprendizaje, y asimismo, brindan a los profesores herramientas teóricas para incluir de mejor manera los medios de apoyo tal y como lo son los traductores y diccionarios online. Específicamente, la pragmática se ocupa de los temas y fenómenos que la sintaxis y la semántica por si mismas no logran abarcar, o sea, la relación entre el significado y las estructuras lingüísticas puestas en funcionamiento dentro del discurso por los hablantes en un contexto o situación específica:

La pragmática toma el lenguaje tal y como se manifiesta, es decir, inmerso en una situación comunicativa concreta; la semántica hace abstracción de los usuarios y se centra en la relación entre los signos y los objetos a los que aquellos representan; y la sintaxis atiende sólo a las relaciones existentes entre los signos, prescindiendo de toda alusión a los usuarios o a los significados. (Escandell, 1993: 7)

Como bien lo señala M. Tordesillas (1992: 12-14), en la línea de las escuelas francesas basadas en las teorías de la enunciación y análisis del discurso desarrolladas principalmente por Emile Benveniste ${ }^{2}$, se encuen-

2 Benveniste (1974) trata el proceso de producción lingüística y el papel del sujeto en la generación de los enunciados. 
tran J.C. Anscombre y O. Ducrot, quienes con la teoría de la argumentación en la lengua (TAL) proponen una pragmática semántica que conjunta tanto la descripción lingüística como el análisis de los actos ilocucionarios en el momento de la enunciación. Con la TAL, Anscombre y Ducrot proponen que el sentido de cualquier enunciado está dado en el proceso argumentativo, por lo que este enfoque es muy adecuado para describir cuáles son las ventajas y las desventajas de las diversas herramientas de traducción en línea y a partir de ello, darles un uso más eficaz en la labor con los estudiantes de E/LE. Según Catalina Fuentes Rodríguez la TAL,

Constituye una de las aportaciones más importantes a la reciente pragmática. Junto con la teoría de los actos del habla, la teoría de la relevancia o el análisis del discurso, dibuja un panorama de estudios que intenta explicar la realidad de la comunicación lingüística, el empleo real de la lengua en los mensajes que emitimos. Esta teoría propugna que todo empleo lingüístico es «per se», argumentativo. Es decir, hablamos para convencer y persuadir a los otros de algo. (2007: 7)

Nuestro punto de partida es que, en la comunicación los seres humanos argumentamos todo el tiempo, por lo cual los diversos marcadores del discurso son esenciales para que los aprendices de una lengua extranjera puedan entrar y desarrollar de mejor forma este proceso, y por ello la importancia de especificar cómo los alumnos pueden hacer uso de las herramientas en línea para acelerar aprendizaje y uso efectivo de éstas partículas.

\section{La Teoría de la Argumentación en la Lengua}

Uno de los aspectos centrales de la TAL es el estudio de aquellos elementos de la lengua que anteriormente no habían sido objeto de interés para los estudiosos, como lo son los marcadores discursivos, pues éstos habían sido considerados como secundarios o paralelos dentro de la descripción lingüística. Desde el punto de vista de las concepciones referencialistas del lenguaje, pocos son los vínculos de estos elementos con las representaciones del mundo, por lo que los marcadores discursivos permanecieron en un plano menos importante. En cambio, la TAL pone especial atención a los conectores, operadores y modificadores del discurso -los más tratados por la teoría de la argumentación, son: pero, por tanto, poco 
DICCIONARIOS Y TRADUCTORES EN LÍNEA EN E/LE: UN ANÁLISIS DES DE LA TEORÍA DE LA ARGUMENTACIÓN EN LA LENGUA

y un poco-. Según José Portolés, a partir de los estudios de Ducrot y Anscombre, "Estos elementos ya no se conciben como meros nexos que coordinan o conectan unas oraciones o unas preposiciones con otras, sino como unidades cuya significación está formada por una serie de instrucciones que hacen comprender de un modo determinado la relación semántica entre los miembros conectados" $(1998,72)$. En La argumentación en la len$g u a^{3}$ publicada por primera vez en 1983, Anscombre y Ducrot proponen una postura estructuralista en la que consideran la lengua como un medio para construir discursos en el marco de la actividad argumentativa: "Anscombre y Ducrot mantienen que no se argumenta «con» la lengua, sino «en» la lengua. Son los propios elementos lingüísticos, y no los hechos que pudieran representar, los que condicionan por su significación la dinámica discursiva" (Portolés, 1998: 73). En la primera etapa de la TAL, los autores consideran a la argumentación como un ente distinto de la lengua. Según ellos, esta etapa, "Se refiere simplemente, al poder que tienen las palabras para describir hechos. Por otro lado, la lengua sirve para marcar la existencia de encadenamientos argumentativos en la medida en que posee conectores [...] que situados entre dos enunciados, indican que los hechos mencionados por uno deben hacer que se admitan los mencionados por el otro" (Anscombre y Ducrot, 1994: 195). Por ejemplo:

(1) El equipo ha jugado como nunca, por lo tanto ha ganado la medalla.

(2) El equipo ha jugado como nunca, sin embargo no ha ganado la medalla.

Ejemplos, en los cuales el rol de los conectores es primordial para dirigir las conclusiones a las que se quiere llegar: mientas que en (1) el conector por lo tanto orienta el enunciado a una conclusión positiva (ha ganado la medalla), en (2) el conector sin embargo predispone a una conclusión negativa (no han ganado la medalla). Estos ejemplos muestran un esquema argumentativo del tipo $\mathrm{A} \rightarrow \mathrm{B}$, en el que "Las frases en sí mismas comportan indicaciones sobre las utilizaciones argumentativas de sus enunciados" (Anscombre y Ducrot, 1994: 196). En esta etapa inicial, es fundamental el estudio de los conectores por lo que la función argumentativa de la lengua está dada en la capacidad de los enunciados para describir hechos y son examinados a partir de leyes discursivas.

Ya en la etapa más madura de la TAL, el argumentativismo radical, los marcadores del discurso no sólo exponen el posicionamiento del locutor res-

3 El título original en francés es: L'argumentation dans la langue (Coll. Philosophie et Langage, Pierre Mardaga éditeur, Bruxelles, 1983, 184 p.) 
pecto de lo que comunica o la actitud que éste quiere imponer al interlocutor al que se dirige, sino que construyen la organización que se pretende dar al discurso, sin embargo, para ello, tal consecución requiere un tercer elemento de índole semántico. Citamos a continuación la explicación de O. Ducrot:

The first idea that I want to bring out is that when you have an argumentative string of the type argument-conclusion, there is always a reference to a third term which is distinct from both the argument and the conclusion, and which allows you to bridge the gap from one to the other. S.E. Toulmin called that third term, the warrant. When I say A, so C, I am supposing that there is something, a warrant, which allows me to bridge the gap from A to B (2009: 65).

Por lo tanto, el discurso argumentativo presenta siempre las indicaciones para el uso de determinados topoi en la enunciación: "Para nosotros, la significación de una frase es el conjunto de los topoi cuya aplicación la frase autoriza en el momento en que es enunciada. En una situación dada, optar por enunciar una frase más que otra es elegir la explotación, en esa situación, de ciertos topoi frente a otros" (Anscombre y Ducrot, 1994: 207). Ducrot lo ilustra con el siguiente ejemplo:

(3) It's warm, let's go for a walk

En (3) el topos aplicado es el siguiente: Cuanto más calor hace, es más agradable la caminata. Debido a que la teoría de la argumentación establece que una de las esencias del lenguaje es su gradualidad y en ésta hay escalas, los topoi pueden aparecer en diversas formas, a las que los autores denominan formas tópicas ${ }^{4}$. Dado que los topoi también son comunes y generales, muchos de ellos son reconocidos por el grupo de hablantes que los apela. No obstante, hay ocasiones en las que éstos recurren a la formulación de nuevos topos, los cuales tienen que ser explicitados y justificados para que la comunicación sea efectiva. La noción de topos es de gran

4 Formas tópicas $(+\mathrm{P},+\mathrm{Q})$ y $(-\mathrm{P},-\mathrm{Q})$, que se orientan hacia el mismo sentido, ya sea positivo, negativo o ascendente, descendente; y formas tópicas $(+\mathrm{P},-\mathrm{Q}) \mathrm{y}(-\mathrm{P},+\mathrm{Q})$, que se conectan en sentidos opuestos; en el ejemplo propuesto por Ducrot estas escalas son representadas de la siguiente manera: «It's warm, let's go for a walk» (+warm, +pleasant -warm, -pleasant) y « It isn't warm, let's go for a walk» (+warm, -pleasant -warm, +pleasant). 
DICCIONARIOS Y TRADUCTORES EN LÍNEA EN E/LE: UN ANÁLISIS DES DE LA TEORÍA DE LA ARGUMENTACIÓN EN LA LENGUA

ayuda, pues como bien lo apunta Tordesillas: "La creación del concepto de formas tópicas nos va a permitir la descripción lingüística en sentido estricto, entendido este como la descripción de morfemas y estructuras cuya combinación constituye las frases de la lengua" (1992: 63). Por lo tanto, es fundamental el rol que juegan los marcadores discursivos, los cuales se explican de acuerdo con la TAL de la siguiente manera:

Conectores argumentativos: Como lo vimos en los ejemplos (1) y (2) son los elementos que relacionan dos enunciados dentro de una frase. Los hay de muchos tipos y tienen evidentes funciones argumentativas, pues cumplen diferentes roles dentro del discurso, la más importante de ellas, es que condicionan su continuidad y dirección. Podemos mencionar, de entre muchos, los conectores que marcan conclusión, conectores aditivos, de justificación, contrastivos, sustitutivos, causales-consecutivos, entre otros. Los conectores, son fundamentales para la teoría de la argumentación en la medida en que "Ponen de manifiesto la conexión semántica entre las partes y marcan la orientación pragmática, y concretamente argumentativa, de los componentes de la oración y del texto" (Cuenca, 1995: 27).

Operadores argumentativos: "Un operador argumentativo es una unidad lingüística que aplicada a una unidad conceptual varía sus posibles continuaciones discursivas" (Portolés, 2004: 237). También, señalan Fuentes y Alcaide (2007: 64) que estas unidades funcionan en el discurso para orientar, reforzar, o marcar la suficiencia de un argumento. Algunos ejemplos de estos morfemas operadores son: hasta, incluso, ya, todavía, casi, apenas, poco, un poco, cierto, cierta. En especial, juegan un papel importante al otorgar a un argumento mayor intensidad o bien, tienen la capacidad de mermar su fuerza. Por ejemplo:

(4) Los estudiantes casi han terminado el ciclo escolar. Sus padres están muy contentos.

(5) Los estudiantes apenas han terminado el ciclo escolar. Sus padres están muy enojados.

Tanto en (4) como en (5), los operadores marcan la dirección del discurso hacia rumbos opuestos; apenas, como operador argumentativo tiende a disminuir la fuerza del segmento que argumenta; en cambio, el operador casi coloca en una tendencia positiva al argumento al otorgarle mayor fuerza, lo que determina las conclusiones en (4) y (5).

Orientación argumentativa: Existen en la lengua ciertos elementos que orientan el discurso hacia determinado sentido. Son argumentos coo- 
rientados aquellos que nos llevan a una conclusión acorde al primer elemento de un encadenamiento, como en los siguientes ejemplos:

(6) Elisa es muy buena cocinera, por eso la cena ha estado deliciosa.

En (6), los argumentos están coorientados para afirmar que Elisa ha deleitado a sus invitados con una cena deliciosa, y concluir que ha sido del gusto de los comensales. El conector por eso, reafirma tal dirección en el encadenamiento. Por otro lado, los argumentos antiorientados, presentan una disyuntiva de unos con otros, para determinar cierta conclusión, como se muestra en los siguientes ejemplos:

(7) Elisa no es muy buena cocinera, pero hoy la cena ha estado deliciosa.

En (7) es el segundo segmento el de mayor peso argumentativo y el que determina la conclusión. En (7), el primer argumento se presenta en contra de las habilidades de Elisa en la cocina, sin embargo el conector pero dirige la conclusión al sentido opuesto, que es que la cena ha sido del gusto de los comensales. Para José Portolés, la orientación argumentativa es un claro ejemplo de lo que Anscombre y Ducrot quieren decir cuando afirman que se argumenta «en» la lengua: "En su teoría cualquier enunciado argumenta en el sentido de que condiciona la dinámica discursiva porque ello es inherente a todo significado lingüístico" (2004: 234).

Modificadores realizantes y desrealizantes: Tienen efecto en la fuerza de los argumentos. La característica más interesante de los modificadores, sean realizantes o desrealizantes, es que inciden en el argumento para darle mayor o menor peso. En un enunciado, los operadores y los conectores pueden fungir como modificadores de ambos tipos, aumentando o disminuyendo la fuerza de los argumentos, y marcando la consecución del discurso, también pueden fungir adjetivos, adverbios, así como ciertos sufijos y prefijos. Proponemos una muestra de cómo funcionan estos elementos:

(8) La película me pareció bastante mala.

(9) Las bebidas en este lugar son algo caras.

En (8) bastante aparece en la frase como un modificador realizante, que da fuerza al adjetivo mala aplicado a la película, con lo que se puede orientar a una conclusión como: «No ganará ningún Oscar». Y por último, en (9), el adjetivo caro que califica a las bebidas, pierde fuerza argumentativa al combinarse con algo, guiando de esta manera el discurso a una consecución como por ejemplo: «pero me gusta». En este caso, es interesante que algo, también sirve para fortalecer la continuidad del discurso con un argumento antiorientado como lo es «pero me gusta». Este tipo de modificadores desrealizantes, operan muy bien en situaciones de cortesía. 
DICCIONARIOS Y TRADUCTORES EN LÍNEA EN E/LE: UN ANÁLISIS DES DE LA TEORÍA DE LA ARGUMENTACIÓN EN LA LENGUA

Por lo tanto, en la teoría de la argumentación los significados se van construyendo dentro de la dinámica discursiva, la cual está inscrita esencialmente en el ámbito lingüístico, por lo que los autores pugnan por una pragmática integrada, en la cual se estudie la construcción de significados a través del uso del material lingüístico; si los significados se construyen argumentando y aparecen como enunciados en el proceso discursivo, es fundamental construir una pragmática semántica, que conjunte precisamente la descripción de los significaciones y sus procesos de construcción: "La prosecución, pues, de un discurso no se basa en los hechos aparentemente representados, sino en lo dicho -el uso, por ejemplo, de unos adverbios determinados o de unos cuantificadores y no de otros-. No se argumenta «con» la representación de unos hechos del mundo por medio de enunciados, sino «en» la misma forma lingüística de estos enunciados” (Portolés, 2004, 234).

\section{Los marcadores discursivos en los diccionarios y traductores online}

Desde que el estudio de los marcadores discursivos cobró fuerza en el ámbito lingüístico, una de las tareas más complicadas de realizar ha sido su clasificación. Al respecto, M. A. Martín Zorraquino (2004: 54-55) ha enfatizado que, tomando en consideración la gran variedad de los marcadores discursivos, su clasificación representa todavía un objetivo difícil de alcanzar, y por esta razón, la tarea que el profesor de español tiene al presentar y explicar estos elementos a los estudiantes, es igualmente complicada. Según la autora, esta complejidad recae en el hecho de que, los marcadores discursivos no contienen en sí mismos significados denotativos, ya que su función principal es marcar la posición que el hablante establece respecto al mensaje que emite, o bien, la relación entre los diversos segmentos del discurso.

Por su parte, Martí Sánchez sostiene que, “[...] conectores, operadores y marcadores conversacionales no constituyen tres compartimentos estancos y la clasificación que los distingue es un principio ordenador que resulta en la práctica demasiado rígido, con lo que las dudas y discusión está garantizadas" (2008: 30), y es precisamente por esta razón que, según el mismo autor, "No pueden estar, pues, tan claramente delimitados como las categorías gramaticales; ya que su fundamento es un contenido y una 
función no ocasionales, pero si dependientes de un contexto, con un cierto grado de formalización que se resiste a ser absoluto" (2008: 30). Así pues, podemos afirmar que no hay, hasta ahora, una clasificación estricta e inamovible para este tipo de partículas, y esta es la razón por la cual, es casi siempre un tema tan complejo de explicar a los estudiantes.

Bajo estas circunstancias se muestra la pertinencia de la TAL, ya que brinda bases más sólidas para presentar estos elementos en su dimensión argumentativa, y con una guía para su uso efectivo, se pueden reforzar las competencias comunicativas en los alumnos. Presentados así, la aparente necesidad de establecer clasificaciones permanentes de estas partículas que resultan confusas para los estudiantes queda en segundo plano, pues más que aprender a qué grupo pertenecen, los estudiantes aprenderán a usarlas en las situaciones comunicativas más variadas. Para ello, los diccionarios y traductores online, deben ser aprovechados por los estudiantes con la guía adecuada que el profesor de E/LE puede proporcionar en este aspecto.

Desde que la aparición y rápido desarrollo de la infinidad de funciones que ofrece el internet, el uso de los traductores online como el traductor de Google, o los diccionarios en línea como Pons, WordReference y Linguee, se ha vuelto muy popular entre los estudiantes de E/LE. Especialmente y a diferencia de los diccionarios tradicionales, los traductores en línea presentaron una innovación especial: La posibilidad de traducir automáticamente enunciados y textos. Sin embargo, tampoco ha tomado mucho tiempo darse cuenta de que, estos traductores muy pocas veces son capaces de traducir el sentido exacto para el que el enunciado es emitido; al respecto W. A. Koza ha señalado que todavía hay mucho que desarrollar en este tipo de programas, especialmente, en lo que a concierne a los marcadores discursivos:

El análisis automático de los marcadores discursivos es una de las tareas fundamentales de la lingüística computacional. La correcta detección de estas construcciones puede ser útil, al resumen y la traducción automáticos, o al análisis sintáctico computacional, entre otras cosas. Uno de los problemas, en este ámbito, está dado por los casos de ambigüedad. (2012: 72)

Muy frecuentemente, los estudiantes se acercan al profesor para preguntar si, el resultado ofrecido por el traductor, en realidad construye un significado preciso, coherente y gramaticalmente correcto, y en ocasiones lo que el traductor da como resultado no corresponde, especialmente, con 
DICCIONARIOS Y TRADUCTORES EN LÍNEA EN E/LE: UN ANÁLISIS DES DE LA TEORÍA DE LA ARGUMENTACIÓN EN LA LENGUA

las funciones argumentativas de determinados marcadores discursivos. Las posibles explicaciones a estas limitaciones parecen tener un mejor fundamento si se explican en términos de la TAL. Precisamente porque esta teoría considera como primordial la función argumentativa de la lengua, desde este enfoque es claro que los traductores de este tipo (en especial el traductor de Google) difícilmente pueden captar el sentido de los enunciados en el contexto en que son emitidos, ya que estos programas traducen mayormente el significado conceptual y no el significado procedural $1^{5}$. Veamos a continuación algunos ejemplos utilizando el traductor de Google para inglés-español y español-inglés. Primero se presenta el enunciado introducido al programa para ser traducido, y a continuación el resultado que el traductor de Google ofrece:

(1) Ya que hoy es día festivo, haremos una excursión a las montañas.

Resultado: Since today is a holiday, we will make a trip to the mountains. ${ }^{6}$

Como podemos ver en (1), el resultado presentado por el programa de Google, efectivamente respeta el carácter procedural del conector ya que. En este caso, el orden sintáctico está invertido, pues la oración subordinada de tipo causal para la que funciona el conector ya que, se encuentra al principio de la frase. No obstante, el traductor de Google efectivamente reconoce que el primer segmento de la frase es consecuencia del segundo segmento. Generalmente, cuando se intenta traducir una frase relativamente pequeña y respetando un orden sintáctico determinado, la función de los conectores como en la frase anterior son bien reconocibles. No obstante, las complicaciones aparecen cuando este orden sintáctico se rompe por medio de la utilización de comas y la organización del discurso se vuelve más compleja; o bien si se utilizan otros conectores con efectos causales, como en el siguiente ejemplo:

5 Desde una perspectiva cognitivista del lenguaje, específicamente desde la teoría de la relevancia, D. Blakemore (1987) establece estas dos categorías, según apunta J. Rajić (2014): "El significado conceptual facilita una información léxica sobre el contenido proposicional, mientras que el significado de procesamiento proporciona las instrucciones sobre cómo nuestro sistema cognitivo debe procesar el significado conceptual. Nombres, verbos, adjetivos y algunos adverbios como libro, leer, hermoso, rápidamente, etc., codifican conceptos, es decir, crean representaciones mentales de objetos, acontecimientos, cualidades, etc.; por el contrario, las expresiones con significado procedimental como sin embargo, por tanto, antes bien, etc. restringen las inferencias de los fragmentos discursivos en los que éstos están empleados, sin afectar a las condiciones de verdad". 
(2) Como hoy es día festivo, haremos una excursión a las montañas. Resultado: As today is a holiday, we will make a trip to the mountains. ${ }^{7}$

En (2), el traductor de Google no puede reconocer que, en esta frase, como funciona semántica y argumentativamente como un conector consecutivo en el discurso, y traduce esta partícula de acuerdo a su función de adverbio en el idioma inglés (as). Otro ejemplo más con el que se puede mostrar los problemas que Google presenta en la identificación de las diversas partículas discursivas es el caso del adverbio tampoco, como vemos en los siguientes ejemplos:

(3) Hoy tampoco ha dejado de llover.

Resultado: It has not stopped raining today. ${ }^{8}$

En (3), tampoco ejerce una función negadora de la acción del verbo que le continúa, y sin embargo, el resultado brindado por Google, no aporta ninguna partícula que pueda ser equiparable con el tampoco del español. En este ejemplo, es particularmente importante la función argumentativa de tampoco, ya que tiene efectos en el significado y la posible consecución del discurso, y que, al no ser tomado en cuenta por el traductor, pierde gran parte de su sentido en el enunciado en inglés. Gracias a tampoco, el discurso podría tener una consecución como la siguiente:

Hoy tampoco ha dejado de llover. Ayer llovió todo el día.

Incluso, las implicaturas que la oración genera con tampoco, no pueden ser generadas con el resultado en inglés, es decir, el enunciado «It has not stopped raining today» no presenta ningún adverbio que indique que otros días ha estado lloviendo también. Sin embargo, en este respecto la TAL no ofrece herramientas suficientes para desarrollar y explicar los efectos e implicaturas de los enunciados, por lo que se requeriría de una teoría de corte cognitivista, como lo es la teoría de la relevancia ${ }^{9}$ para ahondar en este punto.

Por otro lado, en (4) tampoco ejerce la función primordial que convierte al imperativo en negativo, y aunque el traductor de Google logra reconocer que se trata de un imperativo negativo, traduce automáticamente la palabra tampoco, que en el contexto de (4), el adverbio inglés either no hace ningún sentido en la frase.

(4) Linda: Este es el trabajo más difícil del mundo.

Sandra: Tampoco exageres.

$7 \quad$ Consultado el 05.04. 2017

$8 \quad$ Consultado el 07.04.2017

9 La teoría es desarrollada por D. Sperber y D. Wilson en: La relevancia. Comunicación y procesos cognitivos. Madrid, Visor Dis, 1994. 
DICCIONARIOS Y TRADUCTORES EN LÍNEA EN E/LE: UN ANÁLISIS DES DE LA TEORÍA DE LA ARGUMENTACIÓN EN LA LENGUA

Resultado:

Linda: This is the most difficult job in the world.

Sandra: Do not exaggerate either. ${ }^{10}$

Ya por último, en (5) tampoco funge más bien como marcador conversacional. Según Martí Sánchez, estos marcadores "Relacionan y organizan las distintas intervenciones que van produciéndose en la conversación. Son difícilmente eliminables, aunque su presencia no sea muy justificada" (2003: 75), por lo que difícilmente el traductor puede generar un significado equivalente para tampoco en este caso específico:

(5) Héctor: Julia Roberts es la mujer más bella del mundo.

Marina: ¡Tampoco!

Resultado:

Héctor: Julia Roberts is the most beautiful woman in the world.

Marina: ¡Neither! ${ }^{11}$

En, (5), tampoco expresa un desacuerdo respecto al enunciado anterior, y en este caso su significado puede ser equiparado con «no es así» o «no lo creo». Evidentemente, el contexto es determinante cuando se trata de usar los marcadores de tipo conversacional, y en estos casos, es menos probable que el traductor de Google pueda ofrecer una traducción adecuada.

Como hemos visto, aunque es innegable que estas herramientas son útiles para traducir frases muy específicas y fácilmente reconocibles por el programa de traducción automática, el traductor de Google presenta todavía muchas fallas en lo que respecta a los marcadores del discurso, pues éste opera principalmente en el nivel léxico, y por lo tanto traduce un significado asignado a una palabra dentro de determinado orden sintáctico. La deficiencia de una herramienta como esta, vista desde una perspectiva argumentativista como la propuesta por Anscombre y Ducrot, es que no puede abstraer las dinámicas discursivas en las que se generan los significados, por lo que falla en muchas ocasiones al reconocer las diversos roles que puede comportar un marcador discursivo. Es por ello que una de las principales tareas de los profesores e instructores de E/LE, es precisamente, poner al tanto a los estudiantes de estas limitaciones, y mostrarles cómo utilizar esta herramienta de manera más efectiva.

Dado que los marcadores discursivos proporcionan las pautas de direccionamiento del discurso, se puede afirmar que ningún programa de traduc-

$10 \quad$ Consultado el 07.04.2017

11 Consultado el 07.04.2017 
ción automática es infalible, hasta ahora, en lo que concierne a la identificación y traducción exacta de las funciones que realiza determinado marcador en un enunciado; nuevamente hay que señalar que el problema radica en la complejidad de traducir estas partículas, tal y como lo explica J. Rajić (2014): "En el caso de los marcadores discursivos, hay una equivalencia pragmática entre dos lenguas: [...] si entre dos expresiones de distintas lenguas hay alguna equivalencia, ésta se encuentra (aunque no obligatoriamente) en el nivel formal y léxico, pero nunca en el nivel funcional y discursivo".

Cuando los alcances de un traductor en línea, no sean suficientes para cumplir con las necesidades del estudiante, se puede acudir al uso de diccionarios pragmáticos como lo es Linguee, ya que éste ofrece, desde una perspectiva más argumentativista, los diferentes usos de los marcadores discursivos. Si bien Linguee no ofrece la posibilidad de una traducción automática, si ofrece ejemplos de los diversos contextos discursivos en los que se utilizan las palabras y ciertas construcciones verbales. Al revisar la información sobre cuáles son los las palabras o construcciones más buscadas en el diccionario inglés-español y español-inglés, nos hemos encontrado con que, efectivamente, en la lista aparecen un gran número de marcadores discursivos. A continuación mostramos las primeras cincuenta palabras de este listado ${ }^{12}$ :

Búsquedas más frecuentes en español, 1 - 200

¿Qué están traduciendo otras personas en Internet? Vea nuestra lista de búsquedas más frecuentes a continuación.

La lista que acaba de ver fue recientemente calculada en base a consultas de usuarios(as) y se actualiza cada semana.

\begin{tabular}{|c|c|c|c|c|}
\hline 1.: ya que & 2.: $\underline{\sin \text { embargo }}$ & 3.: por lo tanto & 4.: además & 5.: por otro lado \\
\hline $6 .:$ aunque & 7.: es decir & 8.: a partir de & $9 .: \underline{\text { levar a cabo }}$ & $10 .:$ por lo que \\
\hline 11.: asimismo & 12.: no obstante & 13.: por otra parte & 14.: a continuación & 15.: aprovechar \\
\hline 16.: actualmente & 17.: a pesar de & $18 .: \underline{\mathrm{so}}$ & 19.: así como & 20.: mediante \\
\hline 21.: en cuanto a & 22.: realizar & 23.: aportar & 24.: sobre todo & 25.: cumplir \\
\hline 26.: a su vez & 27.: debido a & 28.: en este sentido & 29.: por su parte & 30.: atentamente \\
\hline 31.: presupuesto & 32.: conseguir & 33.: seguimiento & 34.: destacar & $35 .:$ siempre y cuando \\
\hline 36.: tener en cuenta & 37.: de esta manera & 38.: vigente & $39 .:$ por tanto & 40.: posteriormente \\
\hline 41.: con respecto a & 42.: facilitar & 43.: funcionamiento & 44.: empresa & 45.: convocatoria \\
\hline 46.: $\underline{\sin 0}$ & 47.: $\underline{\text { si bien }}$ & 48.: propuesta & 49.: compromiso & $50 .:$ resumen \\
\hline
\end{tabular}

Como podemos ver, una gran parte de las búsquedas realizadas por los usuarios de Linguee, son precisamente partículas de índole instruc-

$12 \mathrm{http} / / / \mathrm{www}$. linguee.mx/spanish-english/topspanish/1-200.html. Consultada el 28.04.2017. 
DICCIONARIOS Y TRADUCTORES EN LÍNEA EN E/LE: UN ANÁLISIS DES DE LA TEORÍA DE LA ARGUMENTACIÓN EN LA LENGUA

cional como los conectores discursivos (ya que, sin embargo, por tanto, por otro lado, etc.) y aparecen también algunos operadores argumentativos (sobre todo). Es evidente también que las palabras de significado conceptual aparecen con menos frecuencia en el listado (empresa, convocatoria, compromiso, resumen, facilitar). Veamos como ejemplo las posibles traducciones que ofrece este diccionario, de acuerdo a las diversas apariciones que el reformulador es decir puede tener en el discurso:

$\begin{array}{ll}\text { Es decir, se debe presumir que el gobi- } & \begin{array}{l}\text { In other words, the } \\ \text { erno divulgará la }\end{array} \\ \text { presumption should be that information } \\ \text { información. } & \text { will be disclosed by the } \\ \text { ediec.org } & \text { government. } \\ \text { ediec.org } & \text { ediec.org } \\ & \text { ediec.org }\end{array}$

Linguee presenta como equivalencias en el idioma inglés principalmente los adverbios in other words, namely y that is to say. Aunque también se pueden obtener ejemplos en los que se puede encontrar correspondencias con otras partículas más ${ }^{13}$ :

Es decir, explican los engranajes de la in- That is, they will explain how the institustitución mas no indican la tion works but won't necessarily conducta que deben observar los Diputa- describe how to behave.

dos.

gopacnetwork.org

gopacnetwork.org gopacnetwork.org

gopacnetwork.org

Es decir, la suma de todos los Thus it is the sum of all the

costes incurridos para completar un caso de costs borne to complete an adoption case.

adopción. hech.net

hcch.net hech.net

hcch.net

No obstante, Linguee muestra también en la misma búsqueda otros ejemplos en los que es decir, no funge como marcador discursivo; por ejemplo, en el siguiente, aparece como parte del contenido de una oración subordinada en el que el significado de decir es primordialmente conceptual:

13 http://www.linguee.mx/espanol-ingles/traduccion/es+decir.html. Consultado el 28.04.2017. 
Liliana Karina Alanís Flores

Una respuesta rápida y sencilla es decir que un acabado con base disolvente es aquél que no contiene agua.

stahl.com

stahl.com
A quick and easy answer is to say that a solvent based finish is one that does not contain any water.

stahl.com

stahl.com

En conclusión, los diccionarios y traductores online son un instrumento que, de ser usados por con la guía adecuada, pueden ser de gran utilidad, tanto en el desarrollo de las competencias comunicativas, discursivas y pragmáticas de los estudiantes de E/LE como en el análisis comparativo que los mismos estudiantes establecen en relación con las lenguas que ya son de su conocimiento. Puesto que los marcadores discursivos permiten una interpretación más acertada de los mensajes, la destreza en el manejo de estos que el estudiante vaya adquiriendo, será un parámetro para cuantificar sus avances en el dominio del idioma. Finalmente, la preparación que los profesores tengan al respecto será clave para lograr estos objetivos, por lo que es importante que ellos presenten interés y la formación adecuada en el tema de los marcadores discursivos:

Para llevar a cabo este tipo de investigación y describir las propiedades semánticas y funcionales de una palabra o expresión gramatical, y de su equivalente en otra lengua, es imprescindible explicar, además de la estructura fonológica, léxica y morfosintáctica, el nivel comunicativo, es decir, dilucidar las características de su uso y las posibilidades de la realización discursiva. (Rajić, 2014)

\section{Referencias bibliográficas}

Anscombre, J. C., Ducrot, O. (1994). La argumentación en la lengua. Madrid: Gredos. Basta, J. (2011). The Role of the Communicative Approach and Cooperative Learning in Higher Education. Facta Universitatis Linguistics and Literature, 9, 2, pp. 125-143.

Benveniste, E. (1974). Problemas de lingüística general II. México: Siglo XXI editores. Blakemore, D. (1987). Semantic constraints on relevance. Oxford: Blackwell.

Cuenca, M. J. (1995). Mecanismos lingüísticos y discursivos de la argumentación. Comunicación, lenguaje y educación, 25, pp. 23-40.

Ducrot, O. (2009). Slovenian Lectures. Introduction into argumentative semantics. Zagar Igor (Ed.). Ljubljana: Pedagoski Institut. 
DICCIONARIOS Y TRADUCTORES EN LÍNEA EN E/LE: UN ANÁLISIS DES DE LA TEORÍA DE LA ARGUMENTACIÓN EN LA LENGUA

Escandell, V. (1993). Introducción a la pragmática. Madrid: Universidad Nacional de Educación a Distancia.

Fuentes Rodríguez, C., Alcaide, E. (2007). La argumentación lingüística y sus medios de expresión. Madrid: Arco libros.

Martí Sánchez, M. (2003). Construcción e interpretación de discursos y enunciados: los marcadores. Madrid: Editorial Edinumen.

Martí Sánchez, M (2008). Los marcadores en Español E/L: conectores discursivos y operadores pragmáticos. Madrid: Arco libros.

Martín Zorraquino, M. A. (2004). El tratamiento lexicográfico de los marcadores del discurso y la enseñanza de ELE. Actas del XV Congreso Internacional de ASELE, pp. 53-70.

Portolés, (1998). La teoría de la argumentación en la lengua y los marcadores del discurso. En M. A. Martín Zorraquino, E. Montolío (coords.), Los marcadores del discurso. Teoría y análisis (pp. 71-92). Madrid: Arco libros.

Portolés, J. (2004). Pragmática para hispanistas. Madrid: Editorial Síntesis.

Rajić, J. (2014). Las teorías pragmáticas y el estudio de los marcadores discursivos en español y en serbio. Disponible en: http://www.fil.bg.ac.rs/wp-content/uploads/obavestenja/iberijske/ehes21/21\%20Jelena\%20Rajic.pdf [11.04.2017]

Savignon, S. J. (1997). Communicative Competence. Theory and Classroom Practice. Texts and Contexts in Second Language Learning. Pennsylvania: McGraw- Hill and The Pennsylvania State University.

Tordesillas, M. (1992). La argumentación: POURTANT en la dinámica argumentativa de los ensayos de Montaigne. (Tesis doctoral no publicada), Facultad de Filología. Universidad Complutense, Madrid.

Liliana Karina Alanís Flores

\section{Rezime}

\section{REČNICI I ONLAJN PREVODIOCI U NASTAVI ŠPANSKOG KAO STRANOG JEZIKA IZ PERSPEKTIVE TEORIJE ARGUMENTACIJE}

U radu se nudi potvrda hipoteze da se pragmatička teorija argumentacije može primeniti u nastavi španskog kao stranog jezika.Sa jedne strane, članak ukazuje na još uvek brojne nedostatke onlajn prevodilaca i rečnika u procesu usvajanja stranog jezika, dok sa druge strane, upravo iz perspektive teorije argumentacije predstavlja mogućnosti primene istih u procesu razvoja komunikativne kompetencije studenta. U radu se akcenat stavlja na potrebu razvoja pragmatičke kompe- 
tencije uz ilustraciju primene elektronske podrške (Google prevodilac i Linguee rečnik) u procesu usvajanja znanja o konektorima i modifikatorima u španskom kao stranom jeziku.

Ključne reči: Španski kao strani jezik, pragmatika, analiza diskursa, konektori i modifikatori, teorija argumentacije u jeziku, komunikativna kompetencija. 